Research Paper

\title{
MiR-34a and miR-203 Inhibit Survivin Expression to Control Cell Proliferation and Survival in Human Osteosarcoma Cells
}

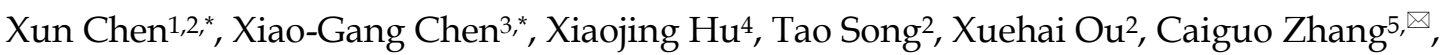 \\ Wentao Zhang, ${ }^{2}$, Chun Zhang, ${ }^{1}$ \\ 1. The second affiliated Hospital of Xi'an Jiaotong University, Xi'an, Shaanxi, China, 710004 \\ 2. Department of Osteology, Xi'an Hong-Hui Hospital affiliated to medical college of Xi'an Jiaotong University, Xi'an, Shaanxi, China, 710054 \\ 3. Department of Orthopaedics, the third affiliated hospital of Zhejiang Chinese Medical University, Hangzhou, China, 310005 \\ 4. Departments of Cardiology, the Ninth affiliated hospital of medical college of Xi'an Jiaotong University, Xi'an, Shaanxi, China, 710054 \\ 5. Department of Biochemistry and Molecular Genetics, University of Colorado School of Medicine, Aurora, CO, USA, 80045 \\ * These authors contribute equally to this work. \\ 凹 Corresponding authors: caiguo.zhang@ucdenver.edu; zhangwentao1975@163.com; zyt1117@126.com
}

() Ivyspring International Publisher. Reproduction is permitted for personal, noncommercial use, provided that the article is in whole, unmodified, and properly cited. See http://ivyspring.com/terms for terms and conditions.

Received: 2015.01.23; Accepted: 2016.03.22; Published: 2016.05.25

\begin{abstract}
Elevated expression of survivin is observed in a number of cancer types, including human osteosarcoma. Few studies have demonstrated that survivin expression levels can be considered an independent predictor of survival for human osteosarcoma patients. However, the underlying molecular mechanisms of survivin in the process of human osteosarcoma carcinogenesis remain unclear. In the current study, we evaluated the biological effects of survivin knockdown on osteosarcoma cell proliferation, colony formation rate, and sensitivity to the chemotherapeutic agent cisplatin. We found that two different osteosarcoma cell lines, U2OS and Saos-2, have relatively higher expression levels of survivin, and specific knockdown of survivin resulted in a number of effects, such as inhibition of cell proliferation, decreased colony formation rate, cell cycle arrest at G2/M phase, induction of apoptosis, and increased sensitivity to cisplatin. In addition, we identified two microRNAs, miR-34a and miR-203, that are aberrantly expressed in human osteosarcoma cells and specifically target survivin by inhibiting its expression, therefore repressing osteosarcoma cell maintenance and proliferation.
\end{abstract}

Key words: Survivin, osteosarcoma, cell proliferation, apoptosis, cisplatin

\section{Introduction}

Osteosarcoma is the most common primary bone malignancy in children and young adults between the ages of 10 and 30 [1,2]. In recent decades, numerous studies have indicated a number of factors, such as negative expression of the retinoblastoma gene $(R b)$ and the tumor suppressor p53, bone dysplasia, and inhibition of apoptosis, that are associated with a high risk of osteosarcoma development [2-8]. Apoptosis is a fundamental process that regulates development and cell differentiation [9, 10]. Dysregulation of apoptosis pathways can lead to a number of diseases, including cancer $[9,10]$. In different organisms, two conserved apoptosis signaling pathways (intrinsic and extrinsic) have been identified to trigger the apoptotic process through the activation of caspases [9-11]. Caspases can be divided into two main classes: initiator and effector caspases [12]. Initiator caspases (caspase-2, -8, -9, and -10) have been suggested to process and activate pro-forms of effector caspases in response to different cell death signals [13]. Effector caspases (caspase- $3,-6$, and -7) play a role in cleaving protein substrates to trigger apoptosis [13]. The apoptosis process can be specifically inhibited by a class of proteins known as inhibitors of apoptosis 
(IAP). The IAPs contain eight members, including Baculoviral IAP Repeat-containing Protein 1-8 (BIRC1-8) in mammals [14-16]. Among these proteins, BIRC1, $-2,-3,-4,-7$, and -8 can directly bind to and inhibit the activation of caspase- $3,-7$, and -9 ; BIRC5 and BIRC6 inhibit apoptosis through two different mechanisms: direct interaction with caspase-3 and -7 , and regulation of cytokine levels [16, 17]. BIRC5 (also known as survivin) is the most important member because of its widely aberrant expression in most types of cancers according to the National Cancer Institute (NCI) cancer drug-screening program [18]. Functional analyses indicate that survivin is widely involved in many processes of tumorigenesis, including tumor cell proliferation, progression, angiogenesis, therapeutic resistance, and poor prognosis [19]. In addition, survivin is also reported to participate in a variety of signaling pathways, such as p53, transforming growth factor (TGF), Wnt, hypoxia, and Notch signaling pathways [15]. Interestingly, the promoter region of the gene encoding survivin contains two cell cycle repressor elements, the cell cycle-dependent element (CDE) and the cell cycle genes homology region (CHR), which control its transcription [20]. A variety of studies demonstrated that survivin expression is most abundant during G2/M phase, thereby participating in the regulation of mitosis by cooperating with a multi-protein complex known as chromosomal passenger complex (CPC) to ensure accurate segregation of sister chromatids [21-25].

In recent years, a great number of microRNAs (miRNAs), such as miR-16, miR-34a, miR-143, miR-150, miR-203, miR-218, miR-320a, miR-494, miR542-3p, and miR-708, have been identified to specifically target to the 3 '-untranslated region (UTR) of survivin. These miRNAs can regulate survivin expression [14], significantly contributing to cancer progression and metastasis [15]. Survivin is highly expressed in osteosarcoma, but the molecular mechanisms involved in its upregulation are unclear. Therefore, we evaluated the effect of survivin downregulation in osteosarcoma cells and tried to identify critical factors regulating survivin expression. Our results indicated that two miRNAs, miR-34a and miR-203, control survivin expression to repress osteosarcoma cell proliferation, colony formation rate, and sensitivity to the chemotherapeutic agent cisplatin.

\section{Materials and Methods}

\section{Cell culture}

The cell lines used in this study included hFOB1.19, U2OS, and Saos-2. Cells were obtained from the American Type Culture Collection (ATCC,
USA) and maintained in DMEM/F-12 medium (1:1, $\mathrm{v} / \mathrm{v}$ ) (Invitrogen, USA) supplemented with 10\% heat-inactivated fetal bovine serum (FBS) (Invitrogen, USA). All cell lines were cultured at $37^{\circ} \mathrm{C}$ in humidified atmosphere containing $95 \%$ air and 5\% $\mathrm{CO}_{2}$ and split twice per week.

\section{Knockdown of survivin expression}

Specific knockdown of survivin expression in hFOB1.19, U2OS, and Saos-2 cells was carried out through a lentiviral system. Both the control vector (pLKO.1-ConshRNA) and human survivin shRNA vectors (pLKO.1-SurshRNA, TRCN0000073720S (S1) and TRCN0000073721 (S2)) were purchased from Sigma (USA). Briefly, the lentiviruses containing either control shRNA or survivin-specific shRNAs (survivin-S1 or survivin-S2) were transfected into hFOB1.19, U2OS, and Saos-2 cells following standard procedures. Transfected cells were selected by puromycin treatment $(5 \mu \mathrm{g} / \mathrm{mL})$ and subjected to the required experiments.

\section{Cell cycle analysis by flow cytometry}

Cell lines $\left(1 \times 10^{6}\right.$ cells) were grown in $100-\mathrm{mm}$ culture dishes, harvested, and washed three times with ice-cold PBS, treated with trypsin, and fixed with nine volumes of $70 \%$ ethanol at $4^{\circ} \mathrm{C}$ for $24 \mathrm{hr}$. The cells were then stained for total DNA content with a solution containing $50 \mu \mathrm{g} / \mathrm{mL}$ propidium iodide (PI), $50 \mu \mathrm{g} / \mathrm{mL}$ RNase, $0.1 \%$ Triton X-100, and $0.1 \mathrm{mM}$ EDTA in PBS for $30 \mathrm{~min}$ at $37^{\circ} \mathrm{C}$. Cell cycle distribution was analyzed by flow cytometry (BD Biosciences, USA).

Flow cytometry analysis of apoptosis was performed using the Annexin V-PE detection kit (BD Biosciences, USA). First, cells were infected with lentiviruses containing either pLKO.1-ConshRNA or pLKO.1-SurshRNAs for $48 \mathrm{hr}$ and then seeded in 6-well plates and washed twice with ice-cold PBS. Cells were then resuspended in $150 \mu \mathrm{L}$ of $1 \mathrm{x}$ binding buffer, followed by the addition of $8 \mu \mathrm{L}$ of Annexin $\mathrm{V}-\mathrm{PE}$ and $8 \mu \mathrm{L}$ of 7-AAD. Cells were incubated for 15 min at $23^{\circ} \mathrm{C}$ in the dark. A minimum of $10^{4}$ stained cells were immediately subjected to flow cytometry.

\section{Western blot analysis}

Whole-cell protein extracts were isolated using a protein extraction buffer containing $150 \mathrm{mM} \mathrm{NaCl}, 10$ $\mathrm{mM}$ Tris (pH7.2), $5 \mathrm{mM}$ ethylenediaminetetraacetic acid (EDTA), 0.1\% Triton X-100, 5\% glycerol, and 1\% sodium dodecyl sulfate (SDS). Equal amounts of proteins were subjected to western blot (WB) analysis. The antibodies used for WB were anti-survivin (rabbit, Abcam, USA), anti-Bcl-2 (mouse, Abcam, USA), anti-Bcl-xL (rabbit, Abcam, USA), anti-caspase-3 (mouse, Abcam, USA), anti-caspase- 8 
(mouse, Cell Signaling Technology, USA), and anti- $\beta$-Actin (mouse, Sigma, USA). Signals from protein blots were recorded using ChemiDoc MP (Bio-Rad, USA). All experiments were replicated three times.

\section{Reverse transcription (RT)-PCR}

Total RNA was extracted using RNAzol reagent (Thermo Fisher Scientific, USA) and treated with RNase-free DNase I (TAKARA, Japan) to remove the genomic DNA. Equal amounts of RNA $(1 \mu \mathrm{g})$ were used for reverse transcription with M-MLV reverse transcriptase (Promega, USA) according to the manufacturer's instructions. The cDNAs were then used for PCR amplification. The primers used for testing the expression of different IAP members in human osteosarcoma cell lines are listed in Table 1.

The PCR program was carried out as follows: $95^{\circ} \mathrm{C}$ for $30 \mathrm{sec}$, followed by 40 cycles of $5 \mathrm{sec}$ at $95^{\circ} \mathrm{C}$, $10 \mathrm{sec}$ at $60^{\circ} \mathrm{C}$, and $30 \mathrm{sec}$ at $72^{\circ} \mathrm{C}$. $\beta$-Actin served as internal control for data normalization. All treatments were performed in triplicate, and gene expression was assessed using the $2^{-\Delta \mathrm{Ct}}$ method.

\section{Analysis of miRNA expression}

Total RNA was extracted and purified using the miRNeasy Mini Kit (QIAGEN, USA) according to the manufacturer's instructions. Briefly, TaqMan MicroRNA Reverse Transcription kit (Thermo Fisher Scientific, USA) was used to generate cDNAs using hairpin primers, which are specific to mature miRNAs. The expression levels of miR-16, miR-34a, $m i R-203, m i R-218$, and $m i R-320 a$ were then measured by real-time PCR using TaqMan MicroRNA Assays (assay ID: 478727, 478048, 478758, 477977, and 478594, respectively, Thermo Fisher Scientific, USA) according to the manufacturer's protocol. RNU6B was chosen as reference gene for miRNA expression. The RT-qPCR program was performed in a Bio-RAD CFX96 system (Bio-Rad, USA) as follows: $95^{\circ} \mathrm{C}$ for 2 min; 40 cycles of $95^{\circ} \mathrm{C}$ for $10 \mathrm{sec}, 56^{\circ} \mathrm{C}$ for $30 \mathrm{sec}, 72^{\circ} \mathrm{C}$ for $30 \mathrm{sec}$; and then followed by $72^{\circ} \mathrm{C}$ for $10 \mathrm{~min}, 4^{\circ} \mathrm{C}$ for $5 \mathrm{~min}$. The relative expression level of miRNA was calculated using the comparative $2^{-\Delta C}$. All reactions

Table. 1 Primers used for RT-qPCR

\begin{tabular}{lll}
\hline Gene & Forward primer & \multicolumn{2}{l}{ Reverse primer $^{\text {survivin }}$} & $5^{\prime}$-TGGCAGCCCTTTCTCAAGGACC-3' & $5^{\prime}$-TCGATGGCACGGCGCACTTTCTCC-3' \\
NIAP & $5^{\prime}$-CTGGTGCTGCCTGAGGTCTTTGGC-3' & $5^{\prime}$-TCTTTCTCTAGGAGCTGGTCACAG-3' \\
XAIP & $5^{\prime}$-CAGCATCAACACTGGCACGAGCAG-3 & $5^{\prime}$-GTACTGACCATTCTGGATACCAGA-3' \\
ILP2 & $5^{\prime}$-TGGGACATGGATGTACTCCGTT-3' & $5^{\prime}$-CCAGAGCTCCCTCAAGTGAACG-3' \\
Bcl-2 & $5^{\prime}$--GGAGATAGTGATGAAGTACATCC-3' & $5^{\prime}$-GCACCGGGCTGAGCGCAGGCCC-3' ; \\
Bcl-xL & $5^{\prime}$-CCGGGAGCTGGTGGTTGACTTT-3' & $5^{\prime}$-GTGGCTCCATTCACCGCGGGGC-3' \\
$\beta$-Actin & $5^{\prime}$-AGAGCTACGAGCTGCCTGAC-3' & $5^{\prime}$-AGCACTGTGTTGGCGTACAG-3' \\
\hline
\end{tabular}

were run in triplicate and presented as the mean \pm S.E.M. Student's $t$-test was used to compare expression levels among different groups.

\section{Cell proliferation assay}

Cell proliferation was determined by IncuCyteTM system (Essen BioScience, USA) according to the manufacturer's instructions. Briefly, after $48 \mathrm{hr}$ of infection, 3000 cells/ well were seeded on 96-well plates and placed into the IncuCyteTM at $37^{\circ} \mathrm{C}$ for 2-3 days. Cell proliferation was measured and analyzed using an IncuCyte phase-only processing module every $8 \mathrm{hr}$. Data represent the mean of three independent experiments.

\section{Colony formation assay}

Cell suspensions treated with trypsin from each group were diluted in DMEM supplemented with $10 \%$ FBS and then seeded onto 6-well plates in complete culture medium containing $0.3 \%$ agar on top of a layer of $0.6 \%$ agar prepared in the same culture medium. The plates were incubated at $37^{\circ} \mathrm{C}$ with $5 \%$ $\mathrm{CO}_{2}$ until the cells had formed sufficiently large colonies (14 days in our assays). The colonies were fixed with $70 \%$ ethanol and stained with $0.5 \%$ crystal violet solution. Plates were photographed and the digital images were manually analyzed to determine the number of colonies.

\section{Statistical analysis}

All experiments were independently replicated at least three times. Data are presented as the mean \pm SD. P-values $<0.001$ were considered statistically significant.

\section{Results}

\section{Human osteosarcoma cells present elevated expression of survivin}

Previous studies showed elevated expression of survivin in osteosarcoma cells [15]. To further confirm this observation, we examined survivin protein and mRNA levels in two human osteosarcoma cell lines, U2OS and Saos-2. Western blot results revealed a significant increase in survivin protein levels in U2OS and Saos-2 cells in comparison to the human osteoblast hFOB1.19 cell line (Figure 1A). At the same time, we also observed high levels of two well-characterized anti-apoptotic proteins (Bcl-2 and Bcl-xL) in U2OS and Saos-2 cells (Figure 1A). Then, we analyzed survivin mRNA levels in the osteosarcoma cells through RT-qPCR to explore whether the 
high protein levels of survivin were due to a mechanism involving increased transcription. Similarly, U2OS and Saos-2 cells also expressed higher levels of survivin, Bcl-2, and Bcl-xL mRNAs than hFOB1.19 cells did but did not express higher levels of other IAP genes, including NIAP, XAIP, and ILP2 (Figures 1B-1G). These results suggest that the expression of survivin in osteosarcoma cells might be regulated at transcriptional level.

\section{Specific knockdown of survivin decreases cell survival rate and suppresses colony formation rate}

Infection of U2OS and Saos-2 cells with lentiviruses containing either non-targeted control shRNA or survivin-specific shRNAs indicated that human osteosarcoma cells in which survivin expression was inhibited grew slowly, implying that survivin expression may positively regulate osteosarcoma cell maintenance and proliferation. As shown in Figures $2 \mathrm{~A}$ and 2B, dramatic downregulation of survivin protein and mRNA levels was detected in U2OS and Saos-2 cells after lentiviral infection when compared to the control. However, it appeared that specific knockdown of survivin did not alter the expression of Bcl-2 and Bcl-xL in both U2OS and Saos- 2 cells. We measured the cell survival rate and found it to be significantly reduced in both U2OS and Saos-2 cells following specific knockdown of survivin in comparison to control cells (Figure 2C).
Interestingly, the cell survival rate at $72 \mathrm{hr}$ after lentiviral infection was lower than that at $48 \mathrm{hr}$, suggesting that osteosarcoma cells expressing less survivin may undergo cell death as a consequence of gene knockdown.

In addition, we also examined colony formation rate in U2OS and Saos-2 cells infected with survivin-specific shRNAs. Downregulation of survivin significantly reduced the number of colonies formed in comparison to the control group (Figures $3 \mathrm{~A}$ and $3 \mathrm{~B}$ ). Quantification of the results is shown in Figure 3C. These data suggest that survivin expression is critical for human osteosarcoma cell proliferation.

\section{Specific knockdown of survivin arrests cell cycle at G2/M phase in human osteosarcoma cells}

Given that survivin is involved in cell cycle regulation, we performed flow cytometry analyses to examine whether downregulation of survivin inhibited cell cycle progression in osteosarcoma cells (Figures 4A-4F). As expected, in osteosarcoma cells expressing lower levels of survivin, upon shRNA transfection, the percentage of cells in $\mathrm{G} 2 / \mathrm{M}$ phase increased dramatically (Figures $4 \mathrm{~B}, 4 \mathrm{C}, 4 \mathrm{E}$, and $4 \mathrm{~F}$ ). These data suggest that cell cycle G2/M arrest may contribute to survivin knockdown-induced growth inhibition in human osteosarcoma cells.

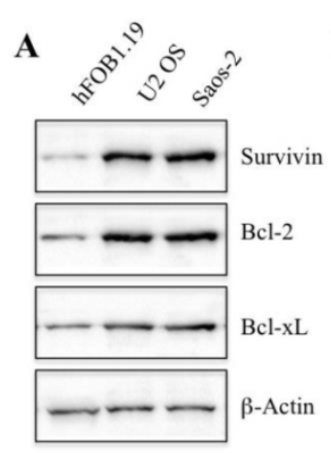

E

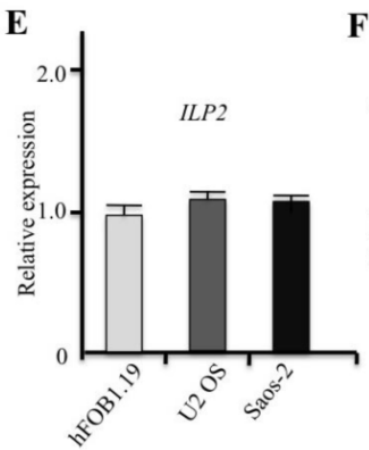

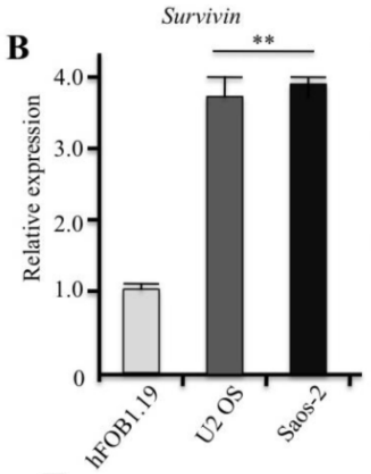

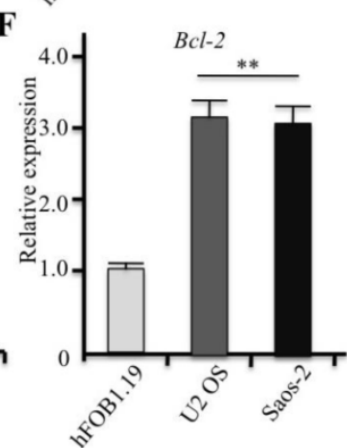

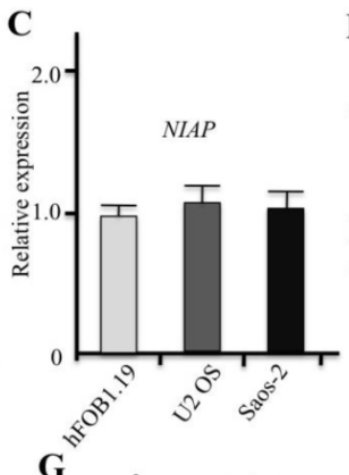
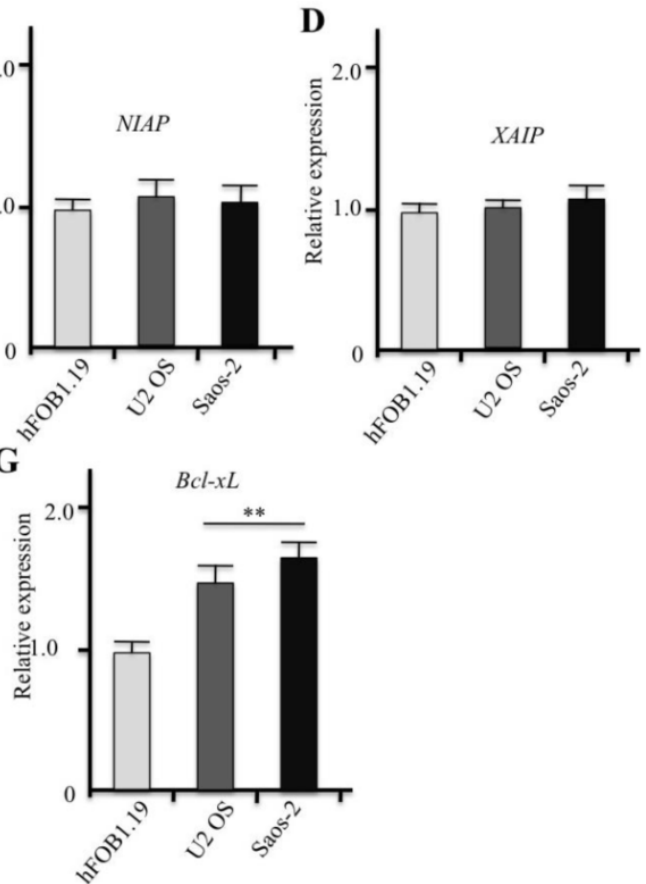

Figure.1. Human osteosarcoma cell lines have elevated expression of survivin. (A) Western blot analyses with specific antibodies directed against survivin, Bcl-2, Bcl-xL, or $\beta$-Actin. (B-G) The mRNA levels of survivin, NIAP, XAIP, ILP2, Bcl-2, and BCl-xL were determined by reverse transcription and RT-qPCR. Expression was normalized against $\beta$-Actin in each cell line, and the resulting ratios in hFOB1.19 cells were arbitrarily defined as 1 -fold. Representative data from three independent experiments are shown. $* * P<0.001$. 
A

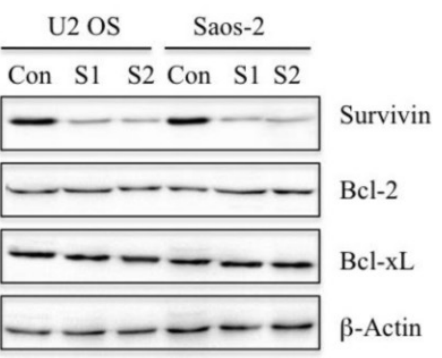

C

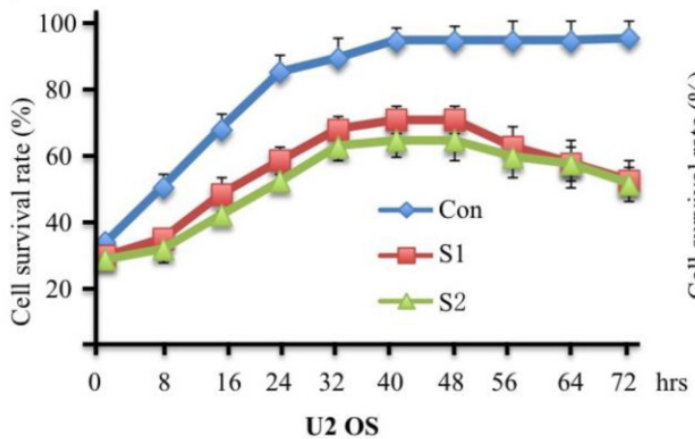

B
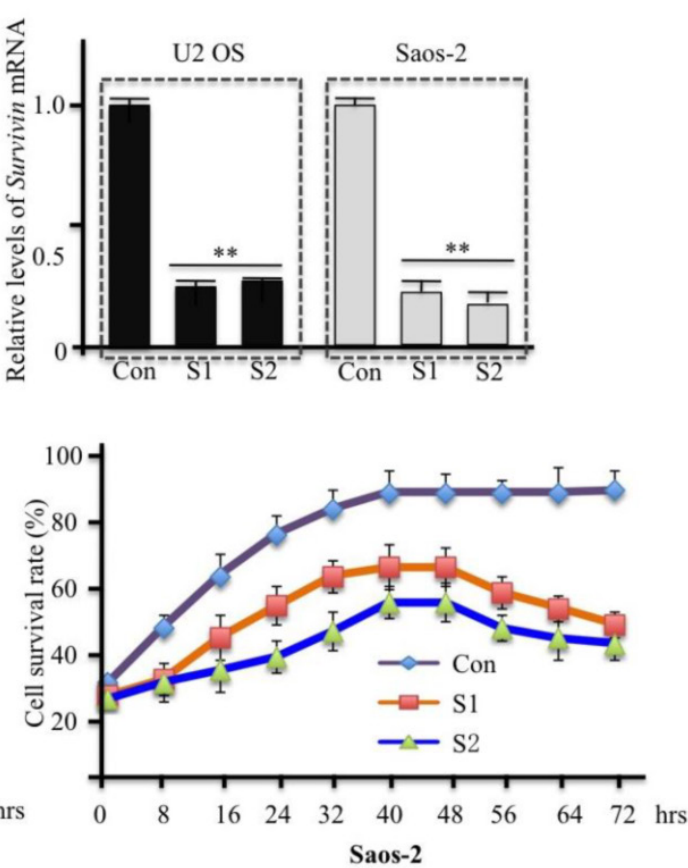

Figure 2. Downregulation of survivin expression by specific shRNAs significantly decreases cell proliferation. U2OS and Saos-2 cells were infected with lentiviruses containing either control shRNA (Con) or survivin-specific shRNAs (S1 and S2). After 48 hr of infection, cells were collected and subjected to western blot analyses with specific antibodies directed against survivin, Bcl-2, Bcl-xL, or $\beta$-actin (A). (B) Survivin mRNA levels were determined by RT-qPCR. Signals were normalized against $\beta$-Actin in each cell line, and the resulting ratios in control cells were arbitrarily defined as 1 -fold. $* * P<0.001$. (C) After $48 \mathrm{hr}$ of lentiviral infection, cells were plated onto 96 -well plates at a density of 5000 cell/well. Cell culture plates were then incubated in a cell culture incubator for 3 additional days. Cell growth curves were generated with the data obtained through a microplate reader at $490 \mathrm{~nm}$ every $8 \mathrm{hr}$. Representative data from three independent experiments are shown.

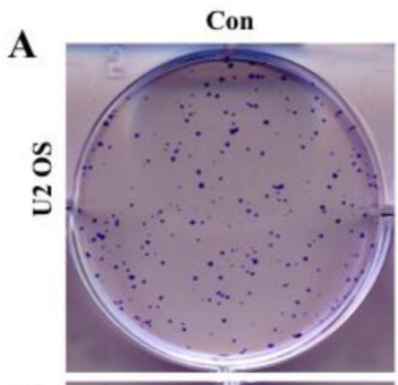

B

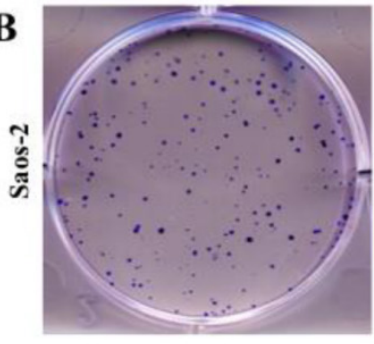

$\mathrm{U} 2 \mathrm{OS}$

C

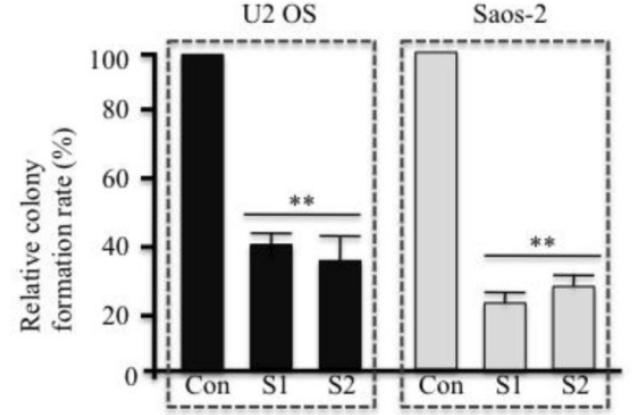

S1
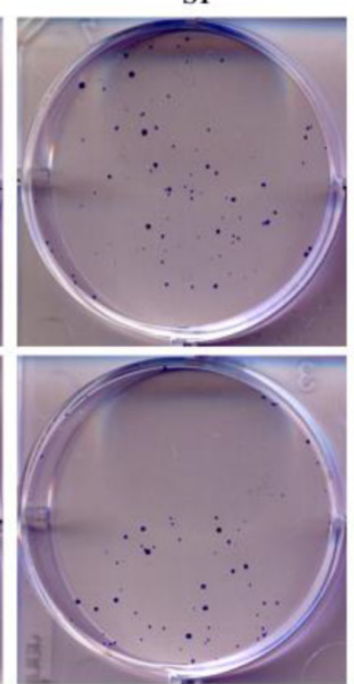

Saos-2
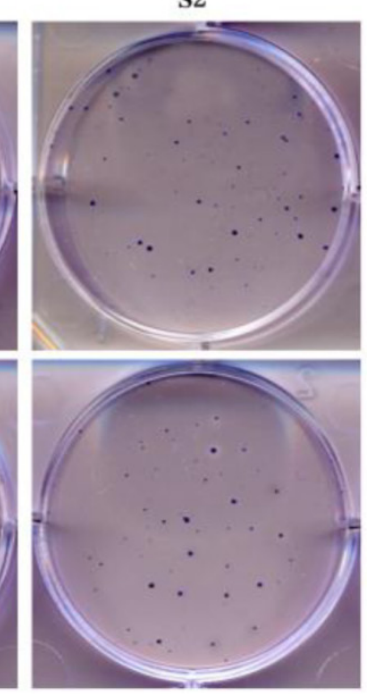

S2 

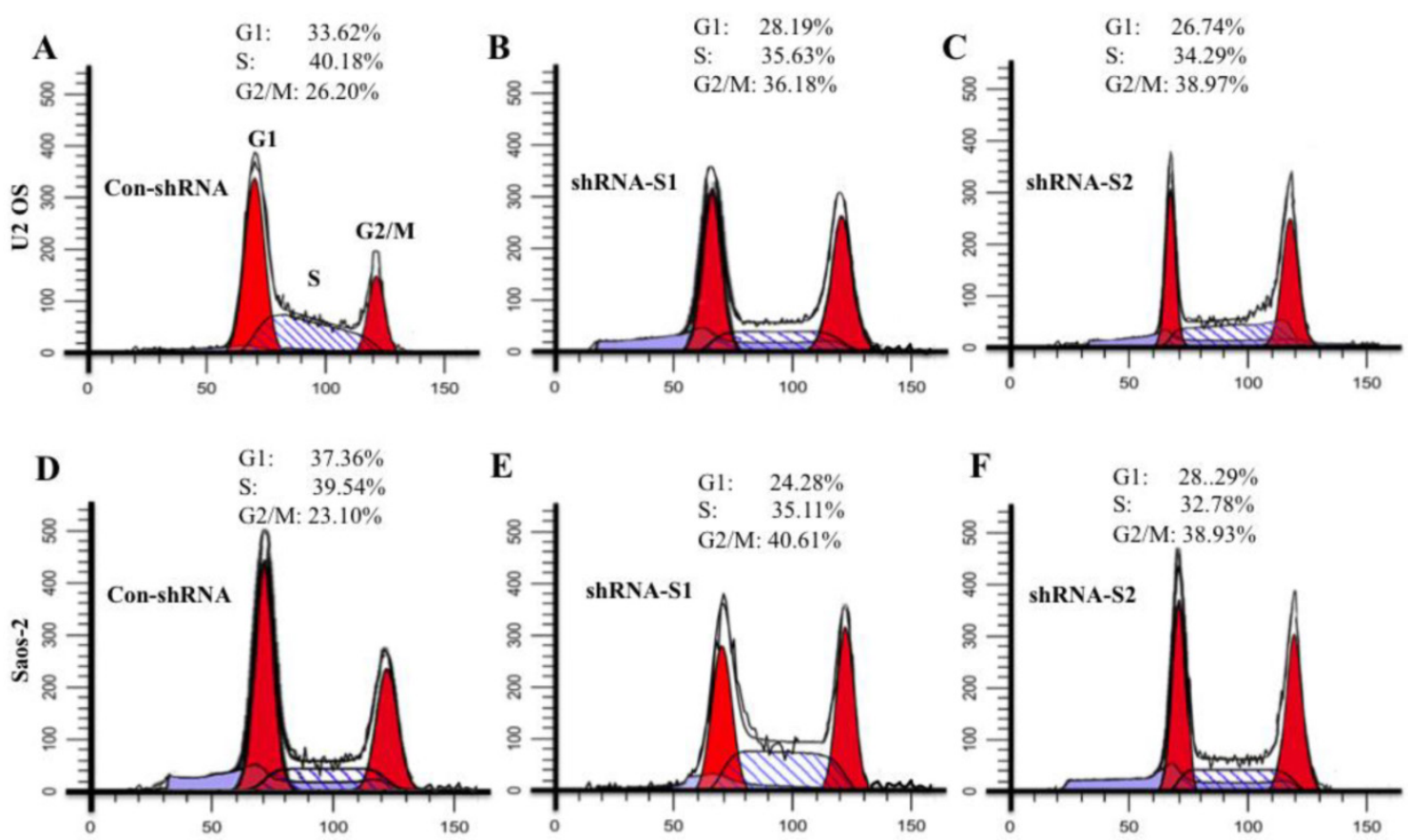

Figure 4. Downregulation of survivin expression by specific shRNAs induces cell cycle arrest at G2/M phase in human osteosarcoma cells. U2OS (A-C) and Saos-2 (D-F) cells were infected with lentiviruses containing either control shRNA (Con) or survivin-specific shRNAs (SI and S2). After 48 hr of infection, cells were collected and subjected to flow cytometry analysis of cell cycle distribution. Representative data from three independent experiments are shown.

\section{Specific knockdown of survivin induces apoptosis in human osteosarcoma cells}

To assess whether the decrease in the cell survival rate resulted from an induction of apoptosis in U2OS and Saos-2 cells, we performed multiparametric flow cytometry by staining the cells with Annexin V-PE and 7-AAD, which is a common method to determine apoptosis. As shown in Figures $5 \mathrm{~A}$ and $5 \mathrm{~B}$, in U2OS and Saos-2 cells infected with survivin-specific shRNAs (S1 and S2) the cell population in Q2 (early apoptosis) and Q3 (late apoptosis) dramatically increased, but not in cells infected with non-targeted control shRNA. Data quantification is shown in Figure 5C. In addition, we also examined the protein levels of caspase- 3 and caspase-8. U2OS and Saos-2 cells infected with survivin-specific shRNAs showed enhanced cleavage of caspase- 8 and caspase-3 (Figure 5D), suggesting that apoptosis in these cells might be caspase-dependent.

\section{Specific knockdown of survivin enhanced cell sensitivity to cisplatin}

To assess the sensitivity of U2OS and Saos-2 cells infected with survivin-specific shRNAs to cisplatin, a widely used chemotherapeutic drug that can ultimately trigger apoptosis, cells were treated with different concentrations of cisplatin $(0,12.5,25,50,75$, 150 , and $300 \mu \mathrm{M})$. A clonogenic survival assay showed that U2OS and Saos-2 cells expressing low levels of survivin were more sensitive to cisplatin-induced cytotoxicity compared with the control groups (Figures 6A and 6B). These results suggested that cisplatin might be a powerful drug against human osteosarcoma.

\section{MiR-34a and miR-203 inhibit survivin expression in human osteosarcoma cells}

Several microRNAs have been reported to directly bind survivin mRNA to regulate its expression. To determine whether these microRNAs also regulated survivin expression in human osteosarcoma cells, we performed RT-qPCR to examine the expression of several important microRNAs, including miR-16, miR-34a, miR-203, $m i R-218$, and miR-320a. As shown in figure 7A, the expression of $m i R-34 a$ and $m i R-203$ dramatically decreased in both U2OS and Saos-2 cells, but no difference was detected in miR-16, $m i R-218$, and $m i R-320 a$ expression. Conversely, U2OS and Saos-2 cells infected with survivin-specific shRNAs presented significantly increased expression of $m i R-34 a$ and $m i R-203$ (Figures 7B and 7C). These data suggest that miR-34a and miR-203 might bind survivin mRNA and negatively regulate its expression in human osteosarcoma cells, repressing cancer cell proliferation and survival. 

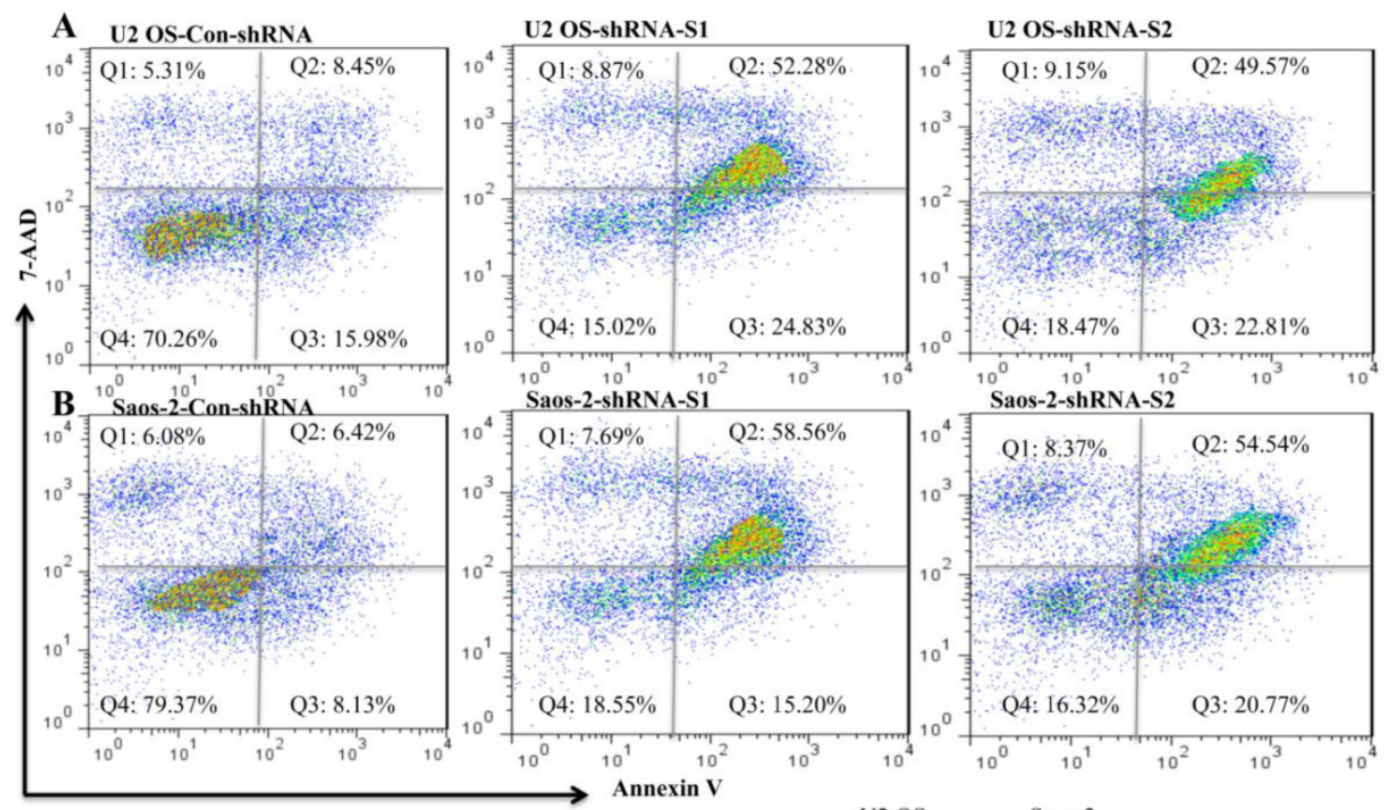

C

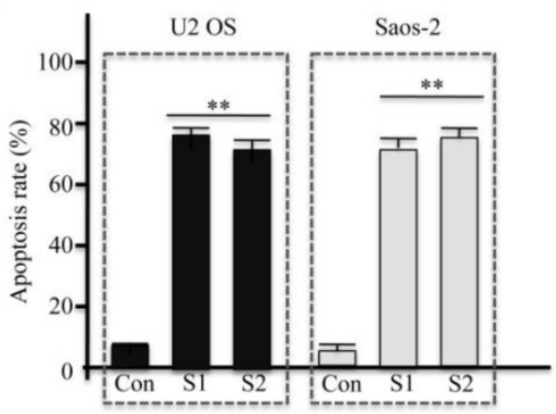

D $\frac{\mathrm{U} 2 \mathrm{OS}}{\mathrm{Con} \quad \mathrm{S} 1 \mathrm{~S} 2} \frac{\mathrm{Saos}-2}{\operatorname{Con} \mathrm{S} 1 \mathrm{~S} 2}$

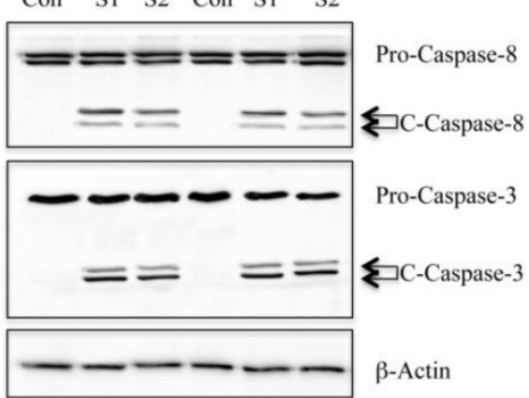

Figure 5. Downregulation of survivin expression by specific shRNAs induces apoptosis in human osteosarcoma cells. U2OS (A) and Saos-2 (B) cells were infected with lentiviruses containing either control shRNA (Con) or survivin-specific shRNAs (S1 and S2). After 48 hr of infection, cells were collected and stained with Annexin V-PE/7-AAD before flow cytometry analysis. Representative results from three independent experiments are shown. (C) Statistical analysis of apoptosis rate in (A) and (B),

$* * P<0.001$. (D) Cells used in (A) and (B) were subjected to western blot analyses with specific antibodies directed against caspase-8 (Pro-Casp-8, full-length caspase-8; C-Casp-8, cleaved caspase-8), caspase-3 (Pro-Casp-3, full-length caspase-3; C-Casp-3, cleaved caspase-3), or $\beta$-Actin.

A

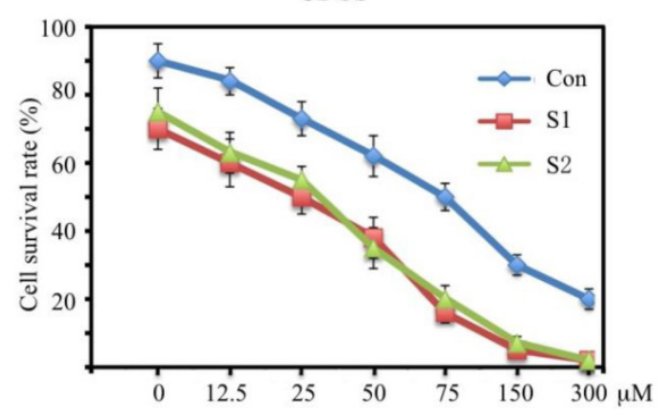

B

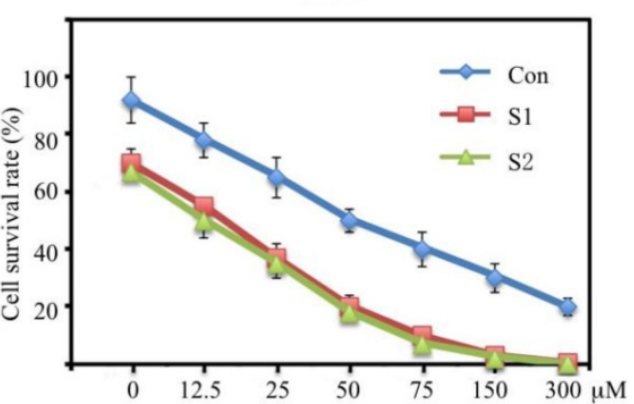

Figure 6. Downregulation of survivin expression by specific shRNAs significantly decreases cell survival rate. U2OS (A) and Saos-2 (B) cells were infected with lentiviruses containing either control shRNA (Con) or survivin-specific shRNAs (S1 and S2). After 48 hr of infection, cells were treated with increasing concentrations of cisplatin $(12.5,25,50,75,150,300 \mu \mathrm{M})$. Cells were harvested $40 \mathrm{~h}$ after treatment (mean $\pm \mathrm{SD}$ ). Cell survival was measured using the MTT assay. Representative data from three independent experiments are shown. 

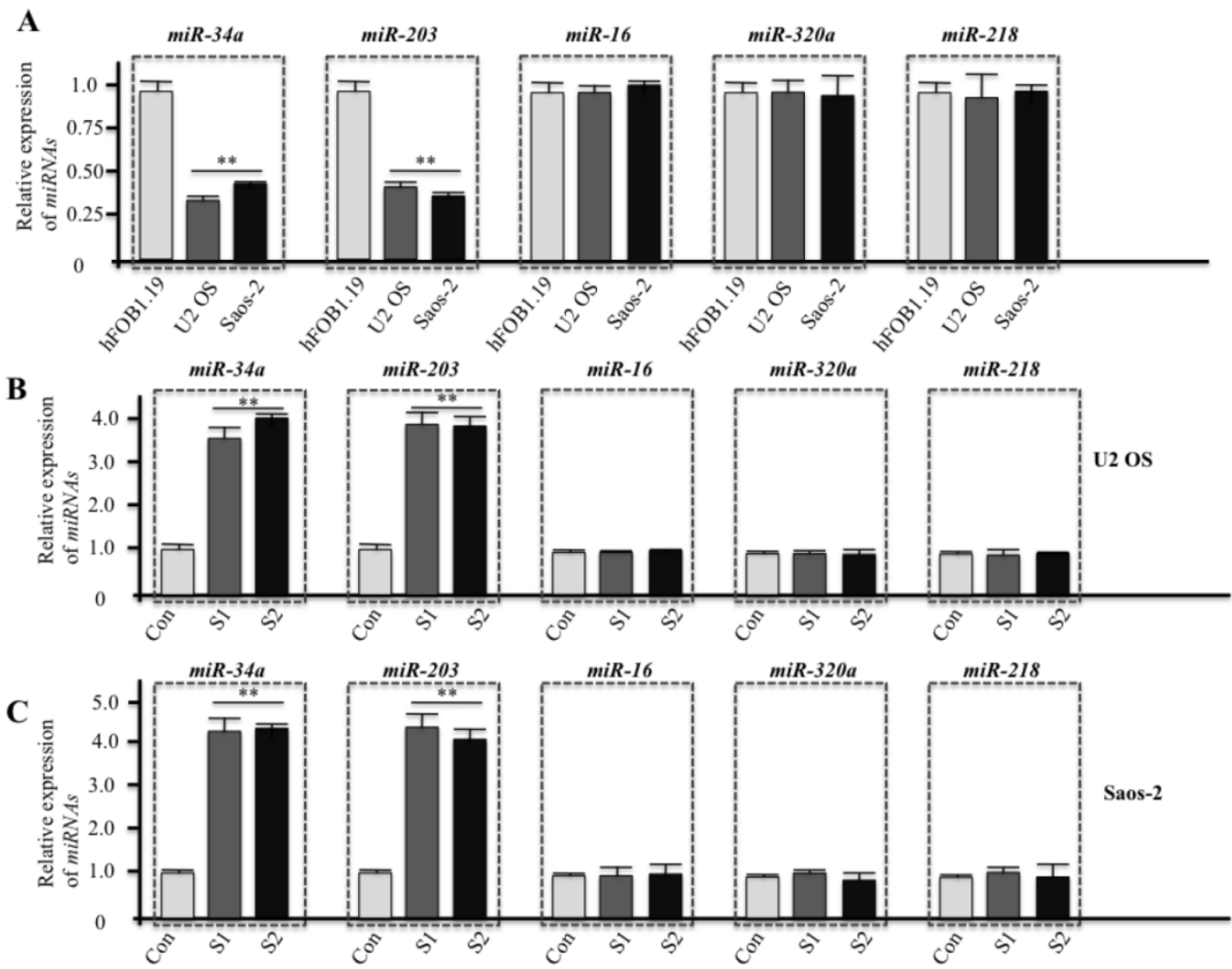

Figure 7. miR-34a and miR-203 regulate survivin expression in human osteosarcoma cells. Cells at normal culture conditions (A) and cells infected with lentiviruses containing either control shRNA (Con) or survivin-specific shRNAs (S1 and S2) (B and C, $48 \mathrm{hr}$ infection) were subjected to total RNA extraction, inclusive of the small RNA fraction. The expression levels of miR-I6, miR-34a, miR-203, miR-2 I8, and miR-320a were measured by RT-qPCR using Taqman miRNA assays. Data were normalized using an internal control (RNU6B). Representative data from three independent experiments are shown. $* * P<0.001$.

\section{Discussion}

Osteosarcoma is one of the most prevalent malignancies in children and young adults. In many cases, it is diagnosed at a very late stage (III or IV). Although advancements in the understanding of carcinogenic mechanisms and therapeutic approaches have significantly increased, the overall survival rates have not improved significantly over the past few years. Thus, it is necessary to further identify factors that control osteosarcoma initiation, progression, and metastasis and to develop new therapeutic strategies to increase the long-term survival of these patients.

In previous research and the present studies, elevated expression of survivin was observed in osteosarcoma cells. In other cancer types, such as breast and lung cancers, increased expression of survivin is associated with cancer cell progression, metastasis, and drug resistance. Thus, inhibition of survivin expression is recognized as a valuable potential strategy for cancer treatment. In our study, we evaluated the biological effects of survivin expression in human osteosarcoma cells and then identified factors involved in regulating survivin expression. Our findings indicate that survivin levels are critical for osteosarcoma cell survival, colony formation, and cell cycle progression. Knockdown of survivin was able to decrease cell proliferation, induce apoptosis and cell cycle arrest, and increase sensitivity to the chemotherapeutic agent cisplatin. These results suggest that survivin is a promising target in the therapy of human osteosarcoma, and cisplatin may be used against osteosarcoma cells. In addition, based on numerous studies revealing that different microRNAs can target survivin to regulate its expression, we performed RT-qPCR to examine the microRNA expression, and found that miR-34a and miR-203 can specifically suppress survivin expression. However, we cannot conclude that miR-34a and miR-203 are the only two microRNAs involved in targeting survivin in human osteosarcoma cells. Our studies only provided a clue about the role of miRNAs in osteosarcoma, and additional analyses using miRNA microarrays or RNA sequencing are necessary to explore the microRNAs targeting survivin in human osteosarcoma cells and clinical specimens. For example, some studies have reported that miR-143 can control cell metastasis in human osteosarcoma cells by targeting matrix metalloprotease-13 [26]. This result further suggests that microRNAs play a critical role in the development of osteosarcoma. In addition, it is also necessary to examine protein and mRNA 
levels of the other known targets of miR-34a and miR-203 (e.g., SIRT1, CDK4, E2F3) to determine if these proteins are also involved in this process. Obviously, microRNAs can target a great number of proteins to regulate various events involved in cancer, such as cancer cell proliferation and metastasis. It remains unknown whether survivin is the only target of miR-34a and miR-203 in human osteosarcoma cells.

In summary, our studies showed that survivin expression in osteosarcoma cells is important for cell proliferation, cell cycle progression, induction of apoptosis, and sensitivity to the chemotherapeutic agent cisplatin. miR-34a and miR-203 can target survivin and negatively regulate its expression. Our findings provide important clues for future studies aimed at unravelling the molecular mechanisms of osteosarcoma carcinogenesis and target survivin in the therapy of human osteosarcoma.

\section{Acknowledgments}

This work was supported by funds from Xi'an Honghui Hospital, Medical College of Xi'an Jiaotong University (YJ2013012).

\section{Competing Interests}

The authors have declared that no competing interest exists.

\section{References}

1. Kundu ZS. Classification, imaging, biopsy and staging of osteosarcoma. Indian J Orthop. 2014; 48: 238-46.

2. Ottaviani G, Jaffe N. The etiology of osteosarcoma. Cancer Treat Res. 2009; 152: 15-32.

3. Lauricella M, D'Anneo A, Giuliano M. et al. Induction of apoptosis in human osteosarcoma Saos-2 cells by the proteasome inhibitor MG132 and the protective effect of pRb. Cell Death Differ. 2003; 10: 930-2.

4. Lamoureux F, Baud'huin M, Rodriguez Calleja L. et al. Selective inhibition of BET bromodomain epigenetic signalling interferes with the bone-associated tumour vicious cycle. Nat Commun. 2014; 5: 3511.

5. Xu Q, Li ZX, Peng HQ. et al. Artesunate inhibits growth and induces apoptosis in human osteosarcoma HOS cell line in vitro and in vivo. J Zhejiang Univ Sci B. 2011; 12: 247-55.

6. Maugg D, Rothenaigner I, Schorpp K. et al. New small molecules targeting apoptosis and cell viability in osteosarcoma. PLoS One. 2015; 10: e0129058.

7. Suzanne M, Steller H. Shaping organisms with apoptosis. Cell Death Differ. 2013; 20: 669-75

8. Ji F, Zhang $\mathrm{H}$, Wang $\mathrm{Y}$, et al. MicroRNA-133a, downregulated in osteosarcoma, suppresses proliferation and promotes apoptosis by targeting Bcl-xL and Mcl-1. Bone. 2013; 56: 220-6.

9. Elmore S. Apoptosis: a review of programmed cell death. Toxicol Pathol. 2007; 35: $495-516$

10. Zhang C, Zhang F. The Multifunctions of WD40 Proteins in Genome Integrity and Cell Cycle Progression. J Genomics. 2015; 3: 40-50.

11. Rao-Bindal K, Kleinerman ES. Epigenetic regulation of apoptosis and cell cycle in osteosarcoma. Sarcoma. 2011; 2011: 679457.

12. Troy CM, Jean YY. Caspases: therapeutic targets in neurologic disease. Neurotherapeutics. 2015; 12: 42-48.

13. McIlwain DR, Berger T, Mak TW. Caspase functions in cell death and disease. Cold Spring Harb Perspect Biol. 2013; 5: a008656.

14. Huang J, Lyu H, Wang J, Liu B. MicroRNA regulation and therapeutic targeting of survivin in cancer. Am J Cancer Res. 2015; 5: 20-31.

15. Chen X, Duan N, Zhang C, Zhang W. Survivin and Tumorigenesis: Molecular Mechanisms and Therapeutic Strategies. J Cancer. 2016; 7: 314-23.

16. Mobahat M, Narendran A, Riabowol K. Survivin as a preferential target for cancer therapy. Int J Mol Sci. 2014; 15: 2494-2516.

17. Saleem M, Qadir MI, Perveen N. et al. Inhibitors of apoptotic proteins: new targets for anticancer therapy. Chem Biol Drug Des. 2013; 82: 243-51.
18. Luk SU, Xue H, Cheng H. et al. The BIRC6 gene as a novel target for therapy of prostate cancer: dual targeting of inhibitors of apoptosis. Oncotarget. 2014; 5: 6896-908.

19. Kusner LL, Ciesielski MJ, Marx A. et al. Survivin as a potential mediator to support autoreactive cell survival in myasthenia gravis: a human and animal model study. PLoS One. 2014; 9: e102231.

20. Muller GA, Engeland K. The central role of CDE/CHR promoter elements in the regulation of cell cycle-dependent gene transcription. FEBS J. 2010; 277: 877-93.

21. Huang J, Lyu H, Wang J, Liu B. Influence of survivin-targeted therapy on chemosensitivity in the treatment of acute myeloid leukemia. Cancer Lett. 2015; 366: 160-72.

22. Beardmore VA, Ahonen LJ, Gorbsky GJ, Kallio MJ. Survivin dynamics increases at centromeres during G2/M phase transition and is regulated by microtubule-attachment and Aurora B kinase activity. J Cell Sci. 2004; 117: 4033-42.

23. Li F, Ambrosini G, Chu EY. et al. Control of apoptosis and mitotic spindle checkpoint by survivin. Nature. 1998; 396: 580-4

24. Tsubaki M, Takeda $\mathrm{T}$, Ogawa N. et al. Overexpression of survivin via activation of ERK1/2, Akt, and NF-kappaB plays a central role in vincristine resistance in multiple myeloma cells. Leuk Res. 2015; 39: 445-52.

25. Chandele A, Prasad V, Jagtap JC. et al. Upregulation of survivin in G2/M cells and inhibition of caspase 9 activity enhances resistance in staurosporine-induced apoptosis. Neoplasia. 2004; 6: 29-40.

26. Osaki M, Takeshita F, Sugimoto Y. et al. MicroRNA-143 Regulates Human Osteosarcoma Metastasis by Regulating Matrix Metalloprotease-13 Expression. Molecular Therapy. 2011; 19: 1123-30. 